\title{
Rotational perturbations in Neveu-Schwarz-Neveu-Schwarz string cosmology
}

\author{
Chiang-Mei Chen* \\ Department of Physics, National Taiwan University, Taipei 106, Taiwan \\ T. Harko ${ }^{\dagger}$ \\ Department of Physics, The University of Hong Kong, Pokfulam, Hong Kong
}

M. K. Mak

Department of Physics, The Hong Kong University of Science and Technology, Clear Water Bay, Hong Kong

(Received 19 December 2000; published 16 April 2001)

\begin{abstract}
First order rotational perturbations of the flat Friedmann-Robertson-Walker metric are considered in the framework of four dimensional Neveu-Schwarz-Neveu-Schwarz string cosmological models coupled with dilaton and axion fields. For the $H$ field we use the solitonic ansatz, assuming that it is a function of time only. The decay rate of rotation depends mainly upon the dilaton field potential $U$. The equation for rotation imposes strong limitations upon the functional form of $U$, restricting the allowed potentials to two: the trivial case $U$ $=0$ and a generalized exponential type potential. In these two models the metric rotation function can be obtained in an exact analytic form in both Einstein and string frames. In the potential-free case the decay of rotational perturbations is governed by an arbitrary function of time while in the presence of a potential the rotation tends rapidly to zero in both Einstein and string frames.
\end{abstract}

DOI: 10.1103/PhysRevD.63.104013

PACS number(s): 04.20.Jb, 04.65.+e, 98.80.-k

\section{INTRODUCTION}

On an astronomical scale, rotation is a basic property of cosmic objects. The rotation of planets, stars, and galaxies inspired Gamow to suggest that the Universe is rotating and the angular momentum of stars and galaxies could be a result of the cosmic vorticity [1]. But even though observational evidence of cosmological rotation has been reported [2-5], it is still a subject of controversy. From the analysis of microwave background anisotropy Collins and Hawking [6] and Barrow, Juszkiewicz, and Sonoda [7] have found some very tight limits on the cosmological vorticity, $T_{\text {obs }}>3 \times 10^{5} T_{H}$, where $T_{\mathrm{obs}}$ is the actual rotation period of our Universe and $T_{H}=(1 \sim 2) \times 10^{10} \mathrm{yr}$ is the Hubble time. Therefore our present day Universe is rotating very slowly, if at all.

From a theoretical point of view in 1949 Gödel [8] gave his famous example of a rotating cosmological solution to the Einstein gravitational field equations. The Gödel metric, describing a dust Universe with energy density $\rho$ in the presence of a negative cosmological constant $\Lambda$ is

$$
d s^{2}=\frac{1}{2 \omega^{2}}\left[-\left(d t+e^{x} d z\right)^{2}+d x^{2}+d y^{2}+\frac{1}{2} e^{2 x} d z^{2}\right] .
$$

In this model the angular velocity of the cosmic rotation is given by $\omega^{2}=4 \pi \rho=-\Lambda$. Gödel also discussed the possibility of a cosmic explanation of the galactic rotation [8]. This rotating solution has attracted considerable interest because the corresponding Universes possess the property of closed timelike curves.

\footnotetext{
*Email address: cmchen@phys.ntu.edu.tw

†Email address: tcharko@hkusua.hku.hk

\#Email address: mkmak@vtc.edu.hk
}

The investigation of rotating and rotating-expanding Universes generated a large amount of literature in the field of general relativity, the combination of rotation with expansion in realistic cosmological models being one of the most difficult tasks in cosmology (see Ref. [9] for a recent review of the expansion-rotation problem in general relativity). Hence rotating solutions of the gravitational field equations cannot be excluded a priori. But this raises the question of why the Universe rotates so slowly. This problem can also be naturally solved in the framework of the inflationary model. Ellis and Olive [10] and Gron and Soleng [11] pointed out that if the Universe came into being as a miniuniverse of Planck dimensions and went directly into an inflationary epoch driven by a scalar field with a flat potential, due to the nonrotation of the false vacuum and the exponential expansion during inflation the cosmic vorticity has decayed by a factor of about $10^{-145}$. The most important diluting effect of the order of $10^{-116}$ is due to the relative density of the rotating fluid compared to the nonrotating decay products of the false vacuum [11]. Inflationary cosmology also ruled out the possibility that the vorticity of galaxies and stars are of cosmic origin.

While scalar field-driven inflationary models resolve many problems of the conventional cosmology, inflationary cosmology is still facing the initial singularity problem. To solve it Gasperini and Veneziano [12] initiated a program, known as pre-big-bang cosmology, which is based on the low-energy effective action resulting from string theory. At the lowest order in the string frame the Neveu-SchwarzNeveu-Schwarz (NS-NS) sector of the four-dimensional string effective action is given by

$$
\hat{S}=\int d^{4} x \sqrt{-\hat{g}} e^{-2 \phi}\left\{\hat{R}+\hat{\kappa}(\nabla \phi)^{2}-\frac{1}{12} \hat{H}_{[3]}^{2}-\hat{U}(\phi)\right\},
$$


where $H_{\mu \nu \lambda}=\partial_{[\mu} B_{\nu \lambda]}$ is the antisymmetric tensor field, $\hat{\kappa}$ is the generalized dilaton coupling constant $(\hat{\kappa}=4$ for string theory) and $\hat{U}(\phi)$ the dilaton potential. Under certain circumstances, this low-energy string action possesses a symmetry property, called scale factor duality, which lets us expect that the present phase of the Universe is preceded by an inflationary pre-big-bang phase.

The possibility of constructing dual solutions is related to the admission of axion fields in the physical model. For spatially homogeneous string cosmologies in $(3+1)$-dimensional low-energy bosonic string theory the most general form of the antisymmetric $H$-field tensor derived from a purely time-dependent potential was derived by Barrow and Kunze [13]. The homogeneous three-form $H$ with a homogeneous potential can have at most three components. The manifestation of duality and asymptotic behavior of the solutions in exact inhomogeneous and anisotropic solutions of low-energy string theory with dilaton and axion fields was considered in Ref. [14]. In general the axiondilaton system is decoupled from the gravitational background due to cylindrical symmetry. The impact of duality upon these models is more subtle than in homogeneous cosmological models, because of the presence of inhomogeneities. For a homogeneous rotating Gödel-type space-time the axion cannot be introduced in the Einstein frame but plays a crucial role in the string frame [15]. Explicit dual solutions can be constructed for each Bianchi space-time, except Bianchi class A types VIII and IX models [16]. Duality transformations for backgrounds with non-Abelian isometries were obtained, by using either a Lagrangian or a Hamiltonian approach, in Ref. [17]. Non-Abelian dualities are of great interest in physical cosmology since some of the most interesting cosmological models have non-Abelian rather than Abelian isometries, important problems such as the flatness cannot even be addressed without considering cosmologies with non-Abelian isometries (Bianchi types V and IX).

By means of the conformal rescaling $g_{\mu \nu}=e^{-2 \phi} \hat{g}_{\mu \nu}$, the action (2) can be transformed to the so-called Einstein frame as

$$
S=\int d^{4} x \sqrt{-g}\left\{R-\kappa(\nabla \phi)^{2}-\frac{1}{12} e^{-4 \phi} H_{[3]}^{2}-U(\phi)\right\},
$$

where $\kappa=6-\hat{\kappa}, U(\phi)=e^{2 \phi} \hat{U}(\phi)$, and $H_{[3]}^{2}$ is the square of the antisymmetric field with respect to the metric $g_{\mu \nu}$. The $H$ field satisfies the integrability condition $\partial_{[\mu} H_{\nu \lambda \alpha]}=0$. Generically, in these types of models the dynamics of the Universe is dominated by massless bosonic fields.

Starting from the actions (2),(3) cosmological models in which the Universe starts out in a cold dilaton-dominated contracting phase, goes through a bounce and then emerges as an expanding Friedmann-Robertson-Walker (FRW) Universe have been explicitly constructed (for a recent and extensive review of pre-big-bang cosmology see Ref. [18]). Exact solutions for the Gödel metric in string theory for the full $O\left(\alpha^{\prime}\right)$ action including both dilaton and axion fields have been obtained by Barrow and Dabrowski [15] who also showed that in low-energy effective string theories Gödel space-times need not contain closed time-like curves. In the absence of the axion field these solutions display a simple relation between the angular velocity $\omega$ of the Gödel Universe and the inverse string tension $\alpha^{\prime}$ of the form $\omega^{2}$ $=1 / \alpha^{\prime}$.

It is the purpose of the present paper to investigate, in the framework of the low energy string effective actions (2),(3), the rotational perturbations of FRW type cosmological models in both Einstein and string frames and to find to what extent the possibility of a rotating and expanding Universe can be incorporated to these types of models. As a general result we find that for a dilaton and axion fields filled Universe the rotational perturbations always decay due to the presence of a dilaton field potential. For a potential-free dilaton field the long-time behavior of rotation is governed by an arbitrary function of time, whose explicit mathematical form cannot be determined in the framework of the first order perturbation theory.

The present paper is organized as follows. In Sec. II we obtain the basic equations describing rotational perturbations of string cosmologies in a flat FRW background. The evolution of rotational perturbations in Einstein frames is considered in Sec. III. The rotation in the string frame is analyzed in Sec. IV. In Sec. V we conclude our results.

\section{GEOMETRY, FIELD EQUATIONS AND CONSEQUENCES}

In four dimensions, every three-form field can be dualized to a pseudoscalar. Thus, an appropriate ansätz for the $H$ field is $\quad H^{\mu \nu \lambda}=(1 / \sqrt{-g}) e^{4 \phi} \epsilon^{\mu \nu \lambda \rho} \partial_{\rho} h, \quad$ where $\quad \epsilon^{\mu \nu \lambda \rho}$ $=-\delta_{[0}^{\mu} \delta_{1}^{\nu} \delta_{2}^{\lambda} \delta_{3]}^{\rho}$ and $h=h(t)$ is the Kalb-Ramond axion field. The gravitational field equations derived from the action (3) are

$$
\begin{aligned}
R_{\mu \nu}-\kappa \partial_{\mu} \phi \partial_{\nu} \phi-\frac{1}{2} e^{4 \phi} \partial_{\mu} h \partial_{\nu} h-\frac{1}{2} g_{\mu \nu} U(\phi) & =0, \\
\nabla^{2} \phi-\frac{1}{\kappa} e^{4 \phi}(\partial h)^{2}-\frac{1}{2 \kappa} \partial_{\phi} U(\phi) & =0, \\
\partial_{\mu}\left(\sqrt{-g} e^{4 \phi} \partial^{\mu} h\right) & =0 .
\end{aligned}
$$

In the Einstein frame the rotationally perturbed metric can be expressed in terms of the usual coordinates in the form [19]

$$
\begin{aligned}
d s^{2}= & -d t^{2}+a^{2}(t)\left[\frac{d r^{2}}{1-k r^{2}}+r^{2}\left(d \theta^{2}+\sin ^{2} \theta d \varphi^{2}\right)\right] \\
& -2 \Omega(t, r) a^{2}(t) r^{2} \sin ^{2} \theta d t d \varphi
\end{aligned}
$$

where $\Omega(t, r)$ is the metric rotation function. Although $\Omega$ plays a role in the "dragging" of local inertial frames, it is not the angular velocity of these frames, except for the special case when it coincides with the angular velocity of the matter fields. 
We assume that rotation is sufficiently slow so that deviations from spherical symmetry can be neglected. For the sake of mathematical simplicity and physical clarity we consider only the case of the flat geometry corresponding to $k=0$. Then to first order in $\Omega$ the gravitational and field equations become

$$
\begin{aligned}
6 \frac{\ddot{a}}{a}+2 \kappa \dot{\phi}^{2}+e^{4 \phi} \dot{h}^{2}-U(\phi) & =0, \\
2 \frac{\ddot{a}}{a}+4 \frac{\dot{a}^{2}}{a^{2}}-U(\phi) & =0, \\
\ddot{\phi}+3 \frac{\dot{a} \dot{\phi}}{a}-\frac{1}{\kappa} e^{4 \phi} \dot{h}^{2}+\frac{1}{2 \kappa} \partial_{\phi} U(\phi) & =0, \\
\ddot{h}+4 \dot{h} \dot{\phi}+3 \frac{\dot{a} \dot{h}}{a} & =0, \\
3 \frac{\dot{a}}{a} \partial_{r} \Omega(t, r)+\partial_{t} \partial_{r} \Omega(t, r) & =0, \\
\partial_{r}^{2} \Omega(t, r)+\frac{4}{r} \partial_{r} \Omega(t, r)-\Omega(t, r)\left(2 \ddot{a} a+4 \dot{a}^{2}\right) & =0 .
\end{aligned}
$$

The axion and dilaton field equations are unperturbed to the first order in $\Omega$. This justifies the assumption of homogeneity in the rotation to first order. Equations (12) and (13) follow from the $\{13\}$ and $\{03\}$ components of the Einstein equation (4), respectively.

Equation (11) can be integrated to give

$$
\dot{h}=C e^{-4 \phi} a^{-3},
$$

with $C \geqslant 0$ an arbitrary constant of integration. Using Eq. (14) the evolution equation of the dilaton field (10) becomes

$$
2 \kappa a^{-3} \frac{d}{d t}\left(a^{3} \dot{\phi}\right)-2 C^{2} e^{-4 \phi} a^{-6}+\frac{d U(\phi)}{d \phi}=0 .
$$

Multiplication of Eq. (15) with $a^{6} \dot{\phi}$ leads to the following first integral of the dilaton field equation:

$$
2 \kappa a^{6} \dot{\phi}^{2}+C^{2} e^{-4 \phi}+2 \int a^{6} \dot{U}(t) d t=2 \kappa \phi_{0},
$$

with $\phi_{0}$ a constant of integration. The elimination of the term $6 \ddot{a} / a$ between the field equations (8) and (9) gives

$$
12 a^{4} \dot{a}^{2}-2 \kappa a^{6} \dot{\phi}^{2}-C^{2} e^{-4 \phi}-2 a^{6} U(\phi)=0 .
$$

Therefore from Eqs. (16) and (17) we obtain the following consistency condition relating the scale factor $a(t)$ to the dilaton field potential:

$$
6 a^{4} \dot{a}^{2}+\int a^{6} \dot{U}(t) d t=a^{6} U(\phi)+\kappa \phi_{0} .
$$

In the present paper we are primarily interested in the evolution of the metric rotation function $\Omega(t, r)$. Equation (12) can be integrated to obtain

$$
\Omega(t, r)=F(r) a^{-3}(t)+G(t),
$$

with $G(t)$ an arbitrary function of time. Therefore from Eqs. (13) and (9) it follows that the function $F(r)$ must obey the equation

$$
\frac{d^{2} F(r)}{d r^{2}}+\frac{4}{r} \frac{d F(r)}{d r}-a^{2}(t) U(\phi)\left[F(r)+G(t) a^{3}(t)\right]=0 .
$$

In order to find the physical interpretation of $G(t)$, we consider the dynamics of a test particle in the perturbed metric (7). The equations of motion are $d u^{\alpha} / d s+\Gamma_{\mu \nu}^{\alpha} u^{\mu} u^{\nu}=0$, where $u^{\mu}$ is the four velocity of test particle and $\Gamma_{\mu \nu}^{\alpha}$ is the Christoffel symbol of the metric. To simplify the calculations we consider only the first order corrections to the metric in $\Omega$ and assume that test particles have small velocities, thus retaining only terms which are linear in velocity. Consequently we obtain

$$
\begin{gathered}
u^{0}=1, \quad \frac{d u^{1}}{d t}=-\frac{2 \dot{a}}{a} u^{1}, \quad \frac{d u^{2}}{d t}=-\frac{2 \dot{a}}{a} u^{2}, \\
\frac{d u^{3}}{d t}=\frac{2 \dot{a}}{a} \Omega+\dot{\Omega}-\frac{2 \dot{a}}{a} u^{3} .
\end{gathered}
$$

By integrating Eq. (21) we find

$$
u^{1}=\frac{u_{0}^{1}}{a^{2}(t)}, \quad u^{2}=\frac{u_{0}^{2}}{a^{2}(t)}, \quad u^{3}=\frac{u_{0}^{3}}{a^{2}(t)}+\frac{F(r)}{a^{3}(t)}+G(t),
$$

with $u_{0}^{i}, i=1,2,3$ constants of integration. In the limit of large $t, \quad a(t) \rightarrow \infty$ and we have $u^{1} \rightarrow 0, u^{2} \rightarrow 0$, and $u^{3}$ $=G(t)$. Therefore $G(t)$ represents the azimuthal velocity of a test particle as measured in the limit of large time (by an observer at an infinite distance in time). At the time $t=t_{\infty}$ the test particle will have shifted in azimuth by $(\Delta \varphi)_{\text {particle }}$ $=\int_{0}^{t_{\infty}} G(t) d t$, having an angular velocity $\omega_{\text {particle }}$ $=(\Delta \varphi)_{\text {particle }} / \Delta t=G\left(t_{\infty}\right)$. Thus $G(t)$ describes the largetime deviations from the radial path due to rotation. Presentday observations impose a strong restriction on the numerical value of the angular velocity of the Universe of the form $G\left(t_{\infty}\right)<10^{-15} \mathrm{yr}^{-1}$ [7]. Hence in realistic cosmological models $G$ must tend to zero in the large time limit.

\section{EVOLUTION OF ROTATIONAL PERTURBATIONS IN THE EINSTEIN FRAME}

The behavior of the rotational perturbations of the isotropic flat FRW cosmological models essentially depends upon the dilaton potential and the function $G(t)$. The form of Eq. (20) governing the spatial part of the metric rotation function imposes strong constraints on the functional form of $U(\phi)$. 
There are two and only two forms of the dilaton field potential which make the rotation equation (20) mathematically consistent.

\section{A. Case I: $U(\phi)=0$}

For $U(\phi) \equiv 0$, Eq. (20) can be immediately integrated to give

$$
F(r)=F_{1}-F_{0} r^{-3},
$$

with $F_{0}, F_{1}$ arbitrary constants of integration. From the field equation (9) we obtain the scale factor $a(t)$ in the form

$$
a(t)=a_{0} t^{1 / 3},
$$

where $a_{0}$ is an arbitrary constant of integration. For this model the deceleration parameter $q$, defined as $q$ $=d H^{-1} / d t-1, H=\dot{a} / a$, is given by $q=2$. The sign of the deceleration parameter shows that the cosmological model inflates or not-negative sign for the inflationary evolution while a positive sign corresponds to standard decelerating expansion. Therefore in the absence of a dilaton potential the cosmological evolution of the axion and dilaton fields filled slowly rotating Universe is noninflationary.

With a vanishing dilaton potential the first integral (16) of the dilaton field equation leads to the following general representation of the conformal transformation factor:

$$
e^{2 \phi(t)}=\sqrt{\frac{C^{2}}{2 \kappa \phi_{0}}} \cosh \left(2 \sqrt{\phi_{0}} \int \frac{d t}{a^{3}}\right) .
$$

With the use of Eq. (24) we find

$$
e^{2 \phi(t)}=\sqrt{\frac{C^{2}}{8 \kappa \phi_{0}}}\left(t^{\sigma}+t^{-\sigma}\right),
$$

where $\sigma=2 \sqrt{\phi_{0}} a_{0}^{-3}$. For the Kalb-Ramond field we obtain

$$
h(t)=h_{0}-\frac{2 \kappa \sqrt{\phi_{0}}}{C} \frac{1}{t^{2 \sigma+1}} .
$$

Equation (18) gives the following consistency condition for the integration constants:

$$
a_{0}^{6}=\frac{3}{2} \kappa \phi_{0} .
$$

With the use of Eq. (28) we can express $\sigma$ as $\sigma=\sqrt{8 / 3 \kappa}$. For string theory $\kappa=2$ and $\sigma=\sqrt{4 / 3}$. For an arbitrary positive $\kappa$ in the range $\kappa \in(0, \infty)$ we have $\sigma \in(\infty, 0)$. Therefore the time evolution of the dilaton and axion fields is entirely determined by the string coupling constant $\kappa$.

The metric rotation function behaves as

$$
\Omega(t, r)=a_{0}^{-3} t^{-1}\left(F_{1}-F_{0} r^{-3}\right)+G(t) .
$$

In the large time limit the behavior of the rotational perturbations of the axion and dilaton fields filled Universe is governed in the Einstein frame by the arbitrary function
$G(t), \quad \lim _{t \rightarrow \infty} \Omega(t, r)=\lim _{t \rightarrow \infty} G(t)$. In order to fix our original assumption of small rotation, we would expect that the function $G(t)$ has the faster or at least equal fall off $1 / t$.

\section{B. Case II: $U(\phi)=U_{0}^{2} a^{-2}(t)$}

The second case in which the rotation equation (20) can be integrated is for a dilaton field potential satisfying the condition

$$
U(\phi)=U_{0}^{2} a^{-2}(t),
$$

with constant $U_{0}$. Then Eq. (20) fixes the arbitrary function $G(t)$ as

$$
G(t) U(\phi) a^{5}(t)=U_{0}^{2} G(t) a^{3}(t)=G_{0} U_{0}^{2},
$$

with $G_{0}$ an arbitrary constant. Consequently $G(t)$ $=G_{0} a^{-3}(t)$. With this choice Eq. (20) becomes

$$
\frac{d^{2} F(r)}{d r^{2}}+\frac{4}{r} \frac{d F(r)}{d r}-U_{0}^{2} F(r)=G_{0} U_{0}^{2} .
$$

Therefore the spatial part of the rotation function is given by

$$
\begin{aligned}
F(r)= & r^{-3}\left[U_{0}^{2} r\left(A e^{U_{0} r}+B e^{-U_{0} r}\right)-U_{0}\left(A e^{U_{0} r}-B e^{-U_{0} r}\right)\right] \\
& -G_{0},
\end{aligned}
$$

where $A$ and $B$ are arbitrary constants of integration. This solution is not regular in the origin $r=0$.

The evolution of the scale factor of the Universe can be obtained from Eq. (9), which with the potential (30) becomes

$$
2 a \ddot{a}+4 \dot{a}^{2}=U_{0}^{2},
$$

and has the general solution

$$
t-t_{0}=\int \frac{a^{2} d a}{\sqrt{K+U_{0}^{2} a^{4} / 4}},
$$

with $K$ an arbitrary constant of integration. Substitution in Eq. (18) gives the consistency condition

$$
6 K=\kappa \phi_{0}
$$

The behavior of the dilaton field $\phi$ is described, as a function of the parameter $a$, by the equation

$$
2 \kappa a^{2}\left(K+\frac{U_{0}^{2}}{4} a^{4}\right)\left(\frac{d \phi}{d a}\right)^{2}+C^{2} e^{-4 \phi}-U_{0}^{2} a^{4}=2 \kappa \phi_{0},
$$

which follows from Eq. (16). The potential cannot be expressed as a function of the dilaton field in terms of elementary functions. In the small-time limit $a \rightarrow 0$ the dilaton field obeys the equation

$$
2 \kappa K a^{2}\left(\frac{d \phi}{d a}\right)^{2}=2 \kappa \phi_{0}-C^{2} e^{-4 \phi},
$$


with the general solution

$$
e^{2 \phi}=\sqrt{\frac{C^{2}}{8 \kappa \phi_{0}}}\left(a^{2 \sqrt{\phi_{0} / K}}+a^{-2 \sqrt{\phi_{0} / K}}\right) \approx \sqrt{\frac{C^{2}}{8 \kappa \phi_{0}}} a^{-2 \sqrt{\phi_{0} / K}} .
$$

The solution of Eq. (35) can be represented in terms of elliptical functions, but in order to have a better physical insight we consider only the solution corresponding to the large time behavior of the model, when the condition $U_{0}^{2} a^{4} / 4 \gg K$ holds with a very good approximation. This is equivalent to taking the arbitrary integration constant $K=0$. Consequently from Eq. (36) we also have $\phi_{0}=0$. Therefore in this limit the Einstein frame time evolution of the scale factor of the rotationally perturbed flat FRW model is given by

$$
a(t)=\frac{U_{0}}{2} t
$$

In this case the deceleration parameter is given by $q=0$. The evolution of the Universe is at the exact limit separating inflationary and noninflationary phases.

The dilaton field equation (16) can be written in the form

$$
\kappa U_{0}^{6} t^{6} \dot{\phi}^{2}+32 C^{2} e^{-4 \phi}-2 U_{0}^{6} t^{4}=0 .
$$

By introducing a new variable $y=e^{-\phi}$, Eq. (41) becomes

$$
\dot{y}^{2}+\frac{32 C^{2}}{\kappa U_{0}^{6}}\left(\frac{y}{t}\right)^{6}-\frac{2}{\kappa}\left(\frac{y}{t}\right)^{2}=0
$$

With the substitution $y=u t$ the general solution of Eq. (42) is given by

$$
\begin{aligned}
& t=t_{0} \frac{[\Delta(u)-1]^{\alpha}}{[\Delta(u)-\sqrt{\kappa / 2}]^{\beta}[\Delta(u)+1]^{\gamma}}, \quad \kappa \neq 2, \\
& t=t_{0}\left(\frac{\Delta(u)-1}{\Delta(u)+1}\right)^{1 / 8} \exp \left[-\frac{1}{4(\Delta(u)-1)}\right], \quad \kappa \neq 2,
\end{aligned}
$$

where $\Delta(u)=\sqrt{1-16 C^{2} u^{4} / U_{0}^{6}}, \quad \alpha^{-1}=4(\sqrt{2 / \kappa}-1), \quad \beta^{-1}$ $=2(2 / \kappa-1), \gamma^{-1}=4(\sqrt{2 / \kappa}+1)$, and $t_{0}$ is a constant of integration.

Therefore the evolution of the dilaton field, dilaton potential and axion field can be represented in the following exact parametric form:

$$
e^{2 \phi}=u^{-2} t^{-2}, \quad U(\phi)=4 t^{-2},
$$

and

$$
\begin{aligned}
h(u) & =\sqrt{\frac{\kappa}{2}} \frac{8 C t_{0}^{2}}{U_{0}^{3}} \int \frac{u^{3}[\Delta(u)-1]^{2 \alpha} d u}{[\Delta(u)-\sqrt{\kappa / 2}]^{2 \beta+1}[\Delta(u)+1]^{2 \gamma}}, \\
\kappa & =2
\end{aligned}
$$

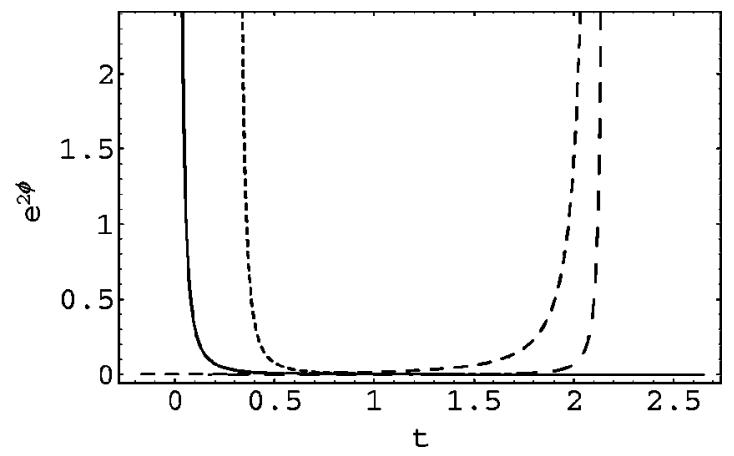

FIG. 1. Einstein frame time evolution of the conformal transformation factor $e^{2 \phi}$ (in units of $10^{6}$ ) in the case of nonvanishing potential dilaton field for different values of the parameter $\kappa: \kappa=1$ (solid curve), $\kappa=3 / 2$ (dotted curve), $\kappa=2$ (short-dashed curve), and $\kappa=3$ (long-dashed curve). We have used the normalizations $16 C^{2} / U_{0}^{6}=1$ and $t_{0}=1$.

$$
\begin{aligned}
h(u)= & \frac{8 C t_{0}^{2}}{U_{0}^{3}} \int u^{3} \frac{[\Delta(u)-1]^{-3 / 4}}{[\Delta(u)+1]^{1 / 4}} \\
& \times \exp \left[-\frac{1}{2[\Delta(u)-1]}\right] d u, \quad \kappa=2,
\end{aligned}
$$

respectively. The dilaton and the axion fields are defined only for values of the parameter $u$ so that $u \leqslant \frac{1}{2} U_{0}^{3 / 2} C^{-1 / 2}$. Therefore during the cosmological evolution the dilaton field satisfies the condition $e^{\phi} \geqslant 2 C^{1 / 2} U_{0}^{-3 / 2} t^{-1}$. For the dilaton potential we obtain $U(\phi) \leqslant U_{0}^{3} C^{-1} e^{2 \phi}$.

The time evolution of the dilaton and Kalb-Ramond axion fields are represented in Figs. 1 and 2, respectively. The dynamics of these fields essentially depend on the string coupling constant $\kappa$. For $\kappa<2$ the dilaton field tends to infinity in the small time limit and for large $t$ decreases rapidly to zero. For $\kappa \geqslant 2$, however, the dilaton field is zero at $t=t_{0}$ $=0$ and is a monotonically increasing function of time. The time variation of the axion shows an opposite dynamics. For $\kappa<2$ the axion field is zero at the initial stages of the evo-

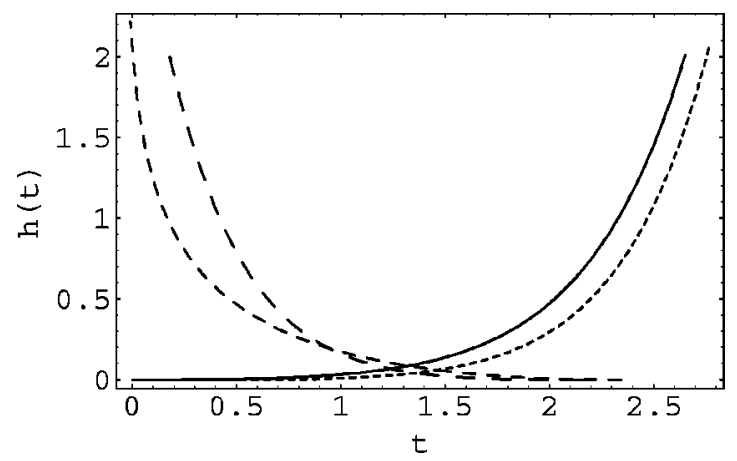

FIG. 2. Einstein frame dynamics of the axion field $h$ (in units of $10^{6}$ ) for the nonvanishing dilaton potential for different values of the string coupling constant $\kappa: \kappa=1$ (solid curve), $\kappa=3 / 2$ (dotted curve), $\kappa=2$ (short-dashed curve), and $\kappa=3$ (long-dashed curve). We have used the normalizations $16 C^{2} / U_{0}^{6}=1$ and $t_{0}=1 / \sqrt{2}$. 
lution of the Universe and then it rapidly increases in time. For $\kappa \geqslant 2$ the axion field tends to infinity for $t=t_{0}=0$ but in the large time limit $h \rightarrow 0$.

The dilaton field potential can be expressed as a function of the dilaton field $\phi$ in the form $U(\phi)=4 u^{2} e^{2 \phi}$. Generally the dilaton field potential can be represented in the form $U(\phi)=g(\phi) e^{2 \phi}$, with $g(\phi)$ a function which does not have, in the present case, an analytical representation. For intervals of time when $u$ can be considered a constant or a slowly varying function of time, the potential-dilaton field dependence is of pure exponential type.

The rotation function is

$$
\begin{aligned}
\Omega(t, r)= & 8 U_{0}^{-2} t^{-3} r^{-3}\left[U_{0} r\left(A e^{U_{0} r}+B e^{-U_{0} r}\right)\right. \\
& \left.-\left(A e^{U_{0} r}-B e^{-U_{0} r}\right)\right] .
\end{aligned}
$$

The time decay of the rotation is inverse proportional to the third power of the time. In the large time limit we have $\lim _{t \rightarrow \infty} \Omega(t, r)=0$. Therefore in the Einstein frame in the presence of an exponential type dilaton field potential there is a rapid decay of the rotational perturbations of the FRW Universe.

For $C=0$, that is, for a constant axion field, which can be chosen, without any loss of generality, to be zero, Eq. (41) gives

$$
e^{2 \phi(t)}=\varphi_{0}^{2} t^{\sqrt{8 / \kappa}}
$$

$\varphi_{0}=$ const. In this case in the large time limit the dilaton field is an increasing function of time.

It is interesting to note that if the dilaton potential is a nonzero constant, $U(\phi) \equiv \Lambda$, then the rotation equation (20) implies $a=$ const. In this case the field equations become inconsistent unless $\Lambda=0$. Therefore the first order rotational perturbations of the FRW cosmological models does not support the existence of a cosmological constant in the Einstein frame.

\section{ROTATIONAL PERTURBATIONS IN THE STRING FRAME}

We consider now the evolution of the rotational metric function in the string frame. In this frame the components of the metric tensor are given by $\hat{g}_{\mu \nu}=e^{2 \phi(t)} g_{\mu \nu}$. If we define a new time variable $\hat{t}$ by means of the transformation $d \hat{t}$ $=e^{\phi(t)} d t$, then in the string frame the line element of the rotationally perturbed metric is given by

$$
\begin{aligned}
d \hat{s}^{2}= & -d \hat{t}^{2}+\hat{a}^{2}(\hat{t})\left[\frac{d r^{2}}{1-k r^{2}}+r^{2}\left(d \theta^{2}+\sin ^{2} \theta d \varphi^{2}\right)\right] \\
& -2 \Omega(\hat{t}, r) \hat{a}^{2}(\hat{t}) e^{-\phi(\hat{t})} r^{2} \sin ^{2} \theta d \hat{t} d \varphi
\end{aligned}
$$

where $\hat{a}^{2}(\hat{t})=e^{2 \phi(\hat{t})} a^{2}(\hat{t})$.

In the case of the potential free dilaton field the string frame cosmological time $\hat{t}$ is defined according to $\hat{t}$ $=\left(C^{2} / 8 \kappa \phi_{0}\right)^{1 / 4} \int \sqrt{t^{\sigma}+t^{-\sigma}} d t$. In the large time limit we obtain $\hat{t} \sim \hat{t}_{0} t^{(\sigma+2) / 2}$, with $\hat{t}_{0}=2\left(C^{2} / 8 \kappa \phi_{0}\right)^{1 / 4} /(\sigma+2)$. The cos- mological time in the string frame is proportional to the cosmological time in the Einstein frame. The conformal transformation factor, also describing the dilaton field evolution, becomes $e^{\phi} \sim\left(C^{2} / 8 \kappa \phi_{0}\right)^{1 / 4}\left(\hat{t} / \hat{t}_{0}\right)^{\sigma /(\sigma+2)}$. For the string frame scale factor we find

$$
\hat{a}(\hat{t}) \sim \hat{a}_{0}\left(\hat{t} / \hat{t}_{0}\right)^{(3 \sigma+2) /(3 \sigma+6)},
$$

where $\hat{a}_{0}=a_{0}\left(C^{2} / 8 \kappa \phi_{0}\right)^{1 / 4}$. The metric tensor component $\hat{g}_{03}$ can be represented as

$$
\begin{aligned}
\hat{g}_{03} \sim & -2\left(\frac{C^{2}}{8 \kappa \phi_{0}}\right)^{-1 / 4}\left[a_{0}^{-1}\left(\frac{\hat{t}}{\hat{t}_{0}}\right)^{(3 \sigma-2) /(3 \sigma+6)}\left(F_{1}-F_{0} r^{-3}\right)\right. \\
& \left.+a_{0}^{2}\left(\frac{\hat{t}}{\hat{t}_{0}}\right)^{(3 \sigma+4) /(3 \sigma+6)} \hat{G}(\hat{t})\right] r^{2} \sin ^{2} \theta
\end{aligned}
$$

The string frame decay of the rotational perturbations is again governed by the arbitrary function $\hat{G}(\hat{t})$, describing in the string frame the large-time deviation of the trajectory of a test particle from the radial path. The Kalb-Ramond field is $h(\hat{t}) \sim h_{0}$ approaching to a constant.

The second situation for which the rotation equation describing the string frame evolution of the slowly rotating Universe has a solution corresponds to the exponential type dilaton potential. We restrict again our analysis to the $K$ $=0$ case only. Then defining the cosmological string frame time as $\hat{t}=\hat{t}_{0}+\int e^{\phi} d t, \hat{t}_{0}=$ const and introducing a new variable $\eta=\sqrt{2 / \kappa} \Delta(u), 0 \leqslant \eta \leqslant \sqrt{2 / \kappa}$, the general solution of the field equations can be represented in the following exact parametric form:

$$
\begin{aligned}
\hat{t}(\eta)= & \hat{t}_{0}+\frac{\kappa \sqrt{C}}{2 U_{0}^{3 / 2}} \int \frac{\eta d \eta}{(1-\eta)\left(1-\kappa \eta^{2} / 2\right)^{5 / 4}} \\
\hat{a}(\eta)= & \sqrt{C / U_{0}}\left(1-\kappa \eta^{2} / 2\right)^{-1 / 4} \\
e^{\phi(\eta)=} & \frac{2 \sqrt{C}}{t_{0} U_{0}^{3 / 2}}\left(1-\kappa \eta^{2} / 2\right)^{-1 / 4} \\
& \times \exp \left[-\frac{\kappa}{4} \int \frac{\eta d \eta}{(1-\eta)\left(1-\kappa \eta^{2} / 2\right)}\right] \\
\hat{U}(\eta)= & \frac{U_{0}^{3}}{C}\left(1-\kappa \eta^{2} / 2\right)^{1 / 2}, \\
h(\eta)= & \frac{\kappa t_{0}^{2} U_{0}^{3}}{8 C} \int \frac{\eta \exp \left[\frac{\kappa}{2} \int \frac{\eta d \eta}{(1-\eta)\left(1-\kappa \eta^{2} / 2\right)}\right]}{1-\eta} d \eta
\end{aligned}
$$

The spatial distribution of the rotation function is described by Eq. (33). The temporal behavior of the string frame metric tensor component $\hat{g}_{03}$ is governed by the function 


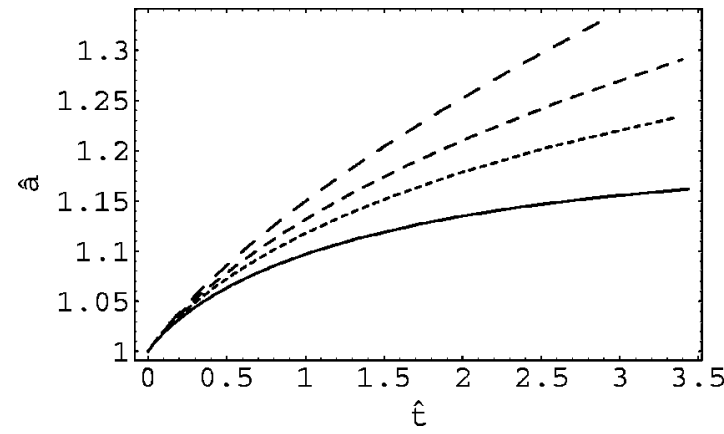

FIG. 3. String frame time evolution of the scale factor $\hat{a}$ in the case of nonvanishing potential dilaton field for different values of the parameter $\kappa: \kappa=1$ (solid curve), $\kappa=3 / 2$ (dotted curve), $\kappa=2$ (short-dashed curve), and $\kappa=3$ (long-dashed curve). We have used the normalizations $\sqrt{C} / 2 U_{0}^{3 / 2}=1$ and $\sqrt{C / U_{0}}=1$, leading to $C$ $=U_{0}=1 / 2$.

$$
\hat{f}(\eta) \sim\left(1-\kappa \eta^{2} / 2\right)^{-1 / 4} \exp \left[-\frac{\kappa}{2} \int \frac{\eta d \eta}{(1-\eta)\left(1-\kappa \eta^{2} / 2\right)}\right] .
$$

In the limit of small $\eta, \quad \eta \rightarrow 0(u \rightarrow 1)$, from Eq. (53) we obtain $\kappa \eta^{2} / 2 \approx 2 U_{0}^{3 / 2} C^{-1 / 2} \hat{t}$. Therefore in the small time limit the scale factor behaves as $\hat{a}(\hat{t}) \approx \sqrt{C / U_{0}}(1$ $\left.-2 U_{0}^{3 / 2} C^{-1 / 2} \hat{t}\right)^{-1 / 4}$. In the string frame and in the presence of the exponential type dilaton potential the slowly rotating Universe starts its evolution from a nonsingular state with $\hat{a}(0)=\sqrt{C / U_{0}} \neq 0$. For the dilaton and axion fields we obtain $e^{\phi(\hat{t})} \approx 2 \sqrt{C / U_{0}^{3}} \exp \left(-\sqrt{U_{0}^{3} / C} \hat{t} / 2\right) \quad$ and $\quad h(\hat{t}) \approx \hat{h}_{0}$ $+\left(U_{0}^{6} t_{0}^{2} / 32 C\right) \exp \left(\sqrt{U_{0}^{3} / C} \hat{t}\right)$, respectively. At the early beginning of the Universe the time behavior of $\hat{g}_{03}$ is given by $\hat{f}(\hat{t}) \approx \exp \left(-2 \sqrt{U_{0}^{3} / C} \hat{t}\right)$.

The time evolutions of the scale factor $\hat{a}$, dilaton field $\phi$, dilaton potential $\hat{U}$, axion field $h$, and $\hat{g}_{03}$ as functions of the string frame cosmological time are represented in Figs. 3-7. The scale factor is represented in Fig. 3 for different $\kappa$. In the string frame the evolution of the Universe starts from a nonsingular state, with the Einstein frame singularity of the scale

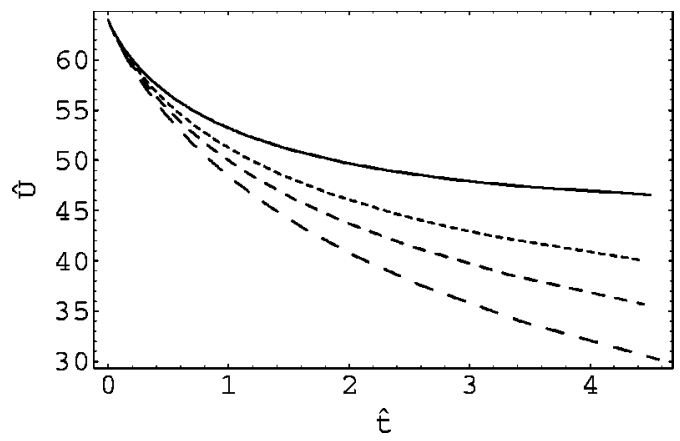

FIG. 4. String frame time dynamics of the dilaton field potential $\hat{U}$ for different values of the parameter $\kappa: \kappa=1$ (solid curve), $\kappa$ $=3 / 2$ (dotted curve), $\kappa=2$ (short-dashed curve), and $\kappa=3$ (longdashed curve). We have used the normalization $U_{0}^{3} / C=1 / 4$.

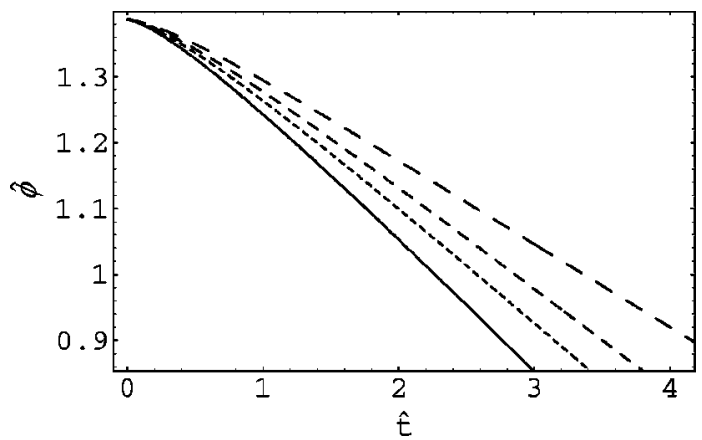

FIG. 5. Variation of the dilaton field $\phi$ as a function of the string frame cosmological time $\hat{t}$ in the case of nonvanishing dilaton potential for different values of the parameter $\kappa$ : $\kappa=1$ (solid curve), $\kappa=3 / 2$ (dotted curve), $\kappa=2$ (short-dashed curve), and $\kappa$ $=3$ (long-dashed curve). We have used the normalizations $t_{0}=1$ and $\sqrt{C} / 2 U_{0}^{3 / 2}=1$.

factor $a(t)=U_{0} t / 2$ removed by the conformal transformation $e^{\phi} a=1 / u$. The dynamics of the dilaton field potential, presented in Fig. 4, shows a monotonically decrease of $\hat{U}$ and in the large time limit $\hat{U} \rightarrow 0$. In Fig. 5 we represented the dynamics of the dilaton field $\phi$. Independently of the values of the string coupling constant $\kappa$, in the large time limit we have $\phi \rightarrow 0$. Hence at the end of the cosmological evolution both the dilaton field and dilaton field potential vanish. But the axion field time variation, presented in Fig. 6, shows a rapid time increase of $h$, with $h \rightarrow \infty$ in the large time limit. Therefore in this model in the large time limit the dynamics of the Universe is governed by the Kalb-Ramond axion field.

In the string frame the time behavior of the rotational perturbations, presented in Fig. 7 is similar to that in the Einstein frame, in the large time limit $\hat{g}_{03} \rightarrow 0$. If in the Einstein frame the time decay of the rotation is given by a power law, being proportional to $t^{-3}$, in the string frame the first order rotational perturbations decay exponentially.

The string frame deceleration parameter $\hat{q}$ is given, as a function of $\eta$, by

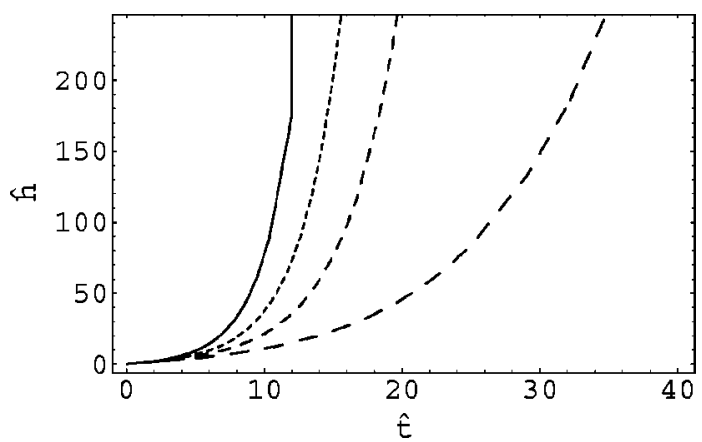

FIG. 6. Time evolution of the axion field $h$ as a function of string frame cosmological time in the case of nonvanishing potential dilaton field for different values of the parameter $\kappa: \kappa=1$ (solid curve), $\kappa=3 / 2$ (dotted curve), $\kappa=2$ (short-dashed curve), and $\kappa$ $=3$ (long-dashed curve). We have used the normalizations $t_{0}$ $=4 \sqrt{2}$ and $\sqrt{C} / 2 U_{0}^{3 / 2}=1$. 


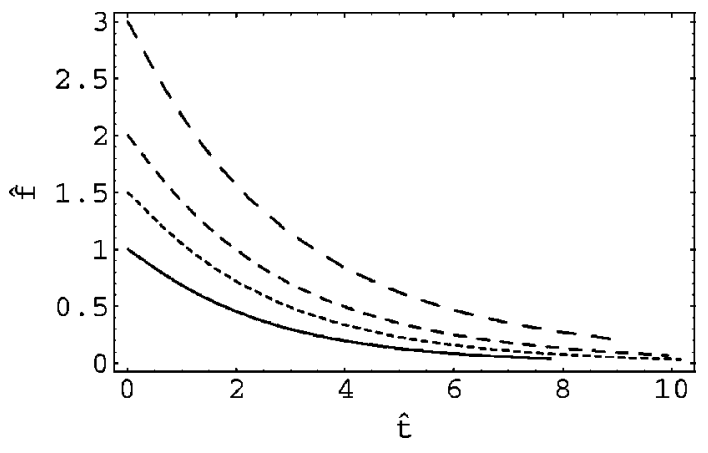

FIG. 7. Time decay of the rotational perturbations $\hat{f}$ as a function of the string frame cosmological time in the case of nonvanishing potential dilaton field for different values of the parameter $\kappa$ : $\kappa=1$ (solid curve), $\kappa=3 / 2$ (dotted curve), $\kappa=2$ (short-dashed curve), and $\kappa=3$ (long-dashed curve). We have used the normalization $\sqrt{C} / 2 U_{0}^{3 / 2}=1$.

$$
\hat{q}(\eta)=\frac{4}{\kappa} \frac{1-\kappa \eta^{2} / 2}{\eta(1-\eta)} .
$$

The time evolution of the deceleration parameter is represented in Fig. 8. The dynamics of $\hat{q}$ is dependent of the string coupling constant $\kappa$. For $\kappa<2$, since values of $\eta>1$ are allowed the Universe ends in the large time limit in an inflationary phase. For $\kappa \geqslant 2$ generally $\hat{q}>0$ and hence the string frame evolution is noninflationary. In this case in the large time limit $\hat{q} \rightarrow 0$ and therefore the Universe ends at the exact limit separating inflationary and noninflationary phases.

For a vanishing axion field the general solution in the string frame can be obtained in an exact form. The string frame cosmological time $\hat{t}$ is related to the Einstein frame cosmological time $t$ by means of the relation $\hat{t}=\left[\varphi_{0} /(\sqrt{2 / \kappa}\right.$ $+1)] t^{\sqrt{2 / \kappa}+1}$. The scale factor has the same behavior as in the Einstein frame, being given by

$$
\hat{a}(\hat{t})=\hat{a}_{0} \hat{t},
$$

with $\hat{a}_{0}=U_{0}(\sqrt{2 / \kappa}+1) / 2$. For this model in the string frame the rotational perturbations

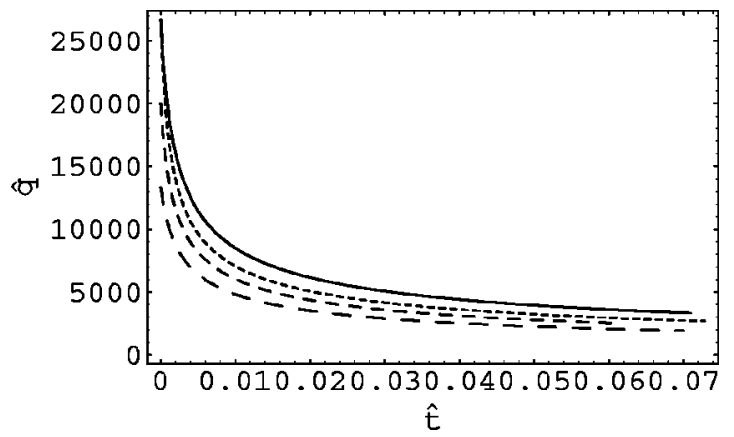

FIG. 8. String frame evolution of the deceleration parameter $\hat{q}$ for different values of the string coupling constant $\kappa: \kappa=1$ (solid curve), $\kappa=3 / 2$ (dotted curve), $\kappa=2$ (short-dashed curve), and $\kappa$ $=3$ (long-dashed curve). We have used the normalization $\sqrt{C} / 2 U_{0}^{3 / 2}=1$.

$$
\begin{aligned}
\hat{g}_{03} \sim \hat{t}^{(\sqrt{2 / \kappa}-1) /(\sqrt{2 / \kappa}+1)}\left[U_{0}^{2} r\left(A e^{U_{0} r}+B e^{-U_{0} r}\right)-U_{0}\left(A e^{U_{0} r}\right.\right. \\
\left.\left.-B e^{-U_{0} r}\right)\right] r^{-1} \sin ^{2} \theta
\end{aligned}
$$

may decay $(\kappa>2)$, increase $(\kappa<2)$, or be independent $(\kappa$ $=2$ ) with respect to the time $\hat{t}$.

\section{DISCUSSIONS AND FINAL REMARKS}

In this paper we have analyzed rotational perturbations of homogeneous flat isotropic FRW in NS-NS string cosmology. To first order in the metric rotation function the field equations reduce to the unperturbed field equations in addition to two equations determining the rotation function $\Omega(t, r)$. The decay of the rotation is basically determined by the dilaton field potential. The field equations impose strong constraints upon the functional form of $U(\phi)$, and except the trivial form $U(\phi)=0$ only one other form is allowed by the mathematical structure of the theory. In the Einstein frame the rotation function can be generally represented as a product of two independent functions, one depending on time and the other on $r$, plus a function $G(t)$ depending on the cosmological time only. From a physical point of view $G(t)$ represents the azimuthal velocity of a test particle as measured by an observer in the infinite future.

If the dilaton field potential is zero, the large-time evolution of the rotational perturbations is determined by the arbitrary function of time $G(t)$ in both Einstein and string frames. Therefore in this case the initial rotation of the Universe may not decay to zero in the large time limit and thus the possibility of a global rotation in the present day Universe is not excluded in this model. On the other hand the arbitrary character of the function $G(t)$ and the absence of a physical mechanism excluding in a natural way rapid latetime rotation of the Universe raises the question if a dilaton potential free pre-big-bang type cosmological model can lead to a correct description of the dynamics of our Universe. This situation is somehow similar to the behavior of the anisotropy in the Bianchi type I space-times in pre-big-bang cosmological models. In the absence of the dilaton field potential a Bianchi type I space-time does not isotropize, the geometry being of Kasner type for all times [20,21]. Therefore in standard pre-big-bang cosmological models with pure dilaton and axion fields neither the initial rotation nor the initial anisotropies can be washed out as a result of the expansionary evolution of the Universe. Of course this conclusion is model and parameters dependent. In the present paper, and also in Ref. [21], a particular model of the pseudoscalar axion field, the so-called solitonic ansatz $h$ $=h(t)$, has been used.

It is possible to consider more general situations with the axion field having a spatial dependence, $h=h(t, x)$ [22]. Four-dimensional FRW and Bianchi type I models for this case have been obtained by Copeland, Lahiri, and Wands [22], by assuming that the $H$-field energy-momentum tensor is homogeneous and isotropic on average. In a flat spacetime the axion field can be decomposed into Fourier modes $h=\Sigma_{q} h_{q}(\eta) \exp \left(i q_{i} x^{i}\right)$ ( $\eta$ is the FRW conformal time coordinate), with $q_{i}$ a spatial comoving three-vector. The solitonic 
ansatz corresponds to $q^{2}=\Sigma_{i} q_{i}^{2} \rightarrow 0$. The equation of motion for axion can also be solved in the case $q^{2} \rightarrow \infty$ (the elementary ansatz). With this choice the $H$ field is decoupled from the dilaton which appears, in the Einstein frame, as a minimally coupled scalar field. The energy-momentum of the axion field is trace-free (of radiation type), given by $-\left\langle{ }^{(h)} T_{0}^{0}\right\rangle$ $=M a^{-4}(t),\left\langle{ }^{(h)} T_{\mu}^{\mu}\right\rangle=3 M a^{-4}(t), M=$ const. The energymomentum tensor of the dilaton field behaves as a stiff fluid, with $-\left\langle{ }^{(\phi)} T_{0}^{0}\right\rangle=\left\langle{ }^{(\phi)} T_{\mu}^{\mu}\right\rangle=N a^{-6}(t), \quad N=$ const. The physical model generally corresponds to the evolution of two noninteracting fluids. But as one can easily check, in this case and in the absence of a dilaton field potential the rotational perturbation equations (12) and (13) are mathematically consistent only for $a=$ const. Therefore first order rotational perturbations do not support the elementary ansatz for the pseudoscalar axion field.

For a nonzero dilaton potential the rotation equation (20) and the field equations completely determine the form of the potential. Therefore neither $U(\phi)$ nor $G(t)$ are arbitrary parameters of the theory. In the Einstein frame the dilaton field potential, can be represented, as a function of time, in a parametric form, with $a$ taken as parameter, as

$$
t-t_{0}=\int \frac{a^{2} d a}{\sqrt{K+U_{0}^{2} a^{4} / 4}}, \quad U(a)=U_{0}^{2} a^{-2} .
$$

This mathematical form of the potential is the only one allowed by the mathematical structure of the field equations. In the limit of small $a, \quad a \rightarrow 0$, the time dependence of the potential is given by $U(t)=U_{0}^{2}(3 \sqrt{K} t)^{-2 / 3}$. In the limit of large $t$, corresponding to large $a$, we obtain $U(t)=4 / t^{2}$.

In the small-time limit the scale factor behaves as $a$ $=(3 \sqrt{K} t)^{1 / 3}$ and the use of Eq. (39) leads to a potentialdilaton field dependence of the exponential form

$$
U(\phi)=\Lambda e^{2 \chi \phi},
$$

where $\Lambda=U_{0}^{2}\left(8 \kappa \phi_{0} / C^{2}\right)^{\sqrt{\phi_{0} / K / 2}}=$ const and $\chi=\sqrt{\phi_{0} / K}$ $=$ const.

The exponential type potentials play an important role in particle physics and cosmology. An exponential potential arises from compactification of the higher-dimensional supergravity in the four-dimensional effective Kaluza-Klein theories or from superstring theories. The moduli fields associated with the geometry of the extra dimensions in string or Kaluza-Klein theories may have effective exponential potentials due to the curvature of the internal spaces or to the interaction of the moduli with form fields on the internal spaces. Exponential potentials can also arise due to the nonperturbative effects such as gaugino condensation (for a discussion of the role of exponential potentials in cosmology see Ref. [20], and references therein). In the large time limit the potential is a generalized exponential one, of the form $U(\phi)=g(\phi) e^{2 \phi}$. The function $g(\phi)$ cannot be expressed in terms of elementary functions. In the string frame and in the same limit the dilaton field potential can be represented as $\hat{U}(\phi)=e^{2 \phi} U(\phi)=4 e^{2 \phi} / t^{2}$.
In the presence of the dilaton field potential there is a rapid decay of the rotational perturbations. The rotation equation (20) fixes not only the form of the potential but also determines the mathematical form of the function $G(t)$, which in the Einstein frame is inversely proportional to the third power of the scale factor. Hence in an expanding Universe the rotational perturbations decrease rapidly, the longtime decay of the rotation being given by a power law in the Einstein and by an exponential term in the string frame. Therefore in the large time limit the azimuthal velocity of a test particle is rigorously zero or very small.

In the spatially isotropic and flat FRW Universe, the effective superstring action is invariant under the discrete $Z_{2}$ transformation $\hat{a} \rightarrow \hat{a}^{(d)}=1 / \hat{a}, \phi \rightarrow \phi^{(d)}=\phi-6 \ln \hat{a}[12]$, that inverts the cosmological scale factor and shifts the value of the dilaton field. This transformation maps an expanding cosmological solution onto a contracting one. But since the theory is also invariant under time reversal $\hat{t}^{(d)}=-\hat{t}$, the contracting solution may be mapped onto a new expanding solution. The Hubble expansion parameter $\hat{H}=d \ln \hat{a} / d \hat{t}$ is invariant under the simultaneous application of scale factor duality and time reversal, but its first derivative changes sign. The new solution will represent a superinflationary cosmology characterized by the condition of the positivity of the first time derivative of the scale factor and of the Hubble parameter and of the second derivative of the scale factor. This is the feature that forms the basis of the pre-big-bang scenario [12]. For the case of the vanishing axion field the string frame scale factor of the Universe is given by Eq. (60), satisfying $d \hat{a} / d \hat{t}=\hat{a}_{0}=$ const $>0$ and $d^{2} \hat{a} / d \hat{t}^{2}=0$. The dual scale factor is $\hat{a}^{(d)}=\hat{a}_{0}^{-1} \hat{t}^{-1}$, with the corresponding Hubble parameter $\hat{H}^{(d)}=-1 / \hat{t}=1 / \hat{t}^{(d)}$. The Hubble parameter has the properties $d \hat{H}^{(d)} / d \hat{t}>0, d \hat{H}^{(d)} / d \hat{t}^{(d)}<0$, while the dual scale factor obeys the restrictions $d \hat{a}^{(d)} / d \hat{t}<0, d \hat{a}^{(d)} / d \hat{t}^{(d)}$ $>0$. Therefore the axion-field free solution with the scale factor linearly increasing in time cannot represent a superinflationary cosmological evolution.

The general physical requirement of the very small (or zero) rotation of the late-time Universe also imposes, in the very early stages of cosmological evolution, the simultaneous presence of both of the axion field $h=h(t)$ and of the dilaton field potential $U(\phi)$.

If the Kalb-Ramond field $h$ is zero, even in the presence of $U(\phi)$, the rotational perturbations in the string frame are governed by the numerical value of the string coupling constant $\kappa$. In this case the Universe is not rotating for large cosmological times only if $\kappa>2$. For string theory $\kappa=2$ and for a zero $h$ field the corresponding dilatonic Universe rotates for all times.

The observational evidence that our Universe is rotating very slowly or at all imposes a major constraint on realistic cosmological models. The first order rotational perturbation theory analyzed in the present paper could be relevant for the understanding of the transient period from a rotating initial state of our Universe to an expansionary one.

\section{ACKNOWLEDGMENTS}

The work of C.M.C. is supported by the Taiwan CosPA project. 
[1] G. Gamow, Nature (London) 158, 549 (1946).

[2] P. Birch, Nature (London) 298, 451 (1982).

[3] P. Birch, Nature (London) 301, 736 (1982).

[4] B. Nodland and J. P. Ralston, Phys. Rev. Lett. 78, 3043 (1997).

[5] R. W. Kühne, Mod. Phys. Lett. A 12, 2473 (1997).

[6] C. B. Collins and S. W. Hawking, Mon. Not. R. Astron. Soc. 162, 307 (1973).

[7] J. D. Barrow, R. Juszkiewicz, and D. H. Sonoda, Mon. Not. R. Astron. Soc. 213, 917 (1985).

[8] K. Gödel, Rev. Mod. Phys. 21, 447 (1949).

[9] Yu. N. Obukhov, in Colloquium on Cosmic Rotation, edited by M. Scherfner, T. Chrobok, and M. Shefaat (Wissenschaft und Technik Verlag, Berlin, 2000), pp. 23-96.

[10] J. Ellis and K. A. Olive, Nature (London) 303, 679 (1983).

[11] Ф. Grøn and H. H. Soleng, Nature (London) 328, 501 (1987).

[12] M. Gasperini and G. Veneziano, Astropart. Phys. 1, 317 (1993)
[13] J. D. Barrow and K. E. Kunze, Phys. Rev. D 55, 623 (1997).

[14] J. D. Barrow and K. E. Kunze, Phys. Rev. D 56, 741 (1997).

[15] J. D. Barrow and M. P. Dabrowski, Phys. Rev. D 58, 103502 (1998).

[16] E. Di Pietro and J. Demaret, Int. J. Mod. Phys. D 8, 349 (1999).

[17] M. Gasperini, R. Ricci, and G. Veneziano, Phys. Lett. B 319, 438 (1993).

[18] J. E. Lidsey, D. Wands, and E. J. Copeland, Phys. Rep. 337, 343 (2000).

[19] V. F. Mukhanov, F. A. Feldman, and R. H. Brandenberger, Phys. Rep. 215, 203 (1992).

[20] C.-M. Chen, T. Harko, and M. K. Mak, Phys. Rev. D 62, 124016 (2000).

[21] C.-M. Chen, T. Harko, and M. K. Mak, Phys. Rev. D 63, 064002 (2001).

[22] E. J. Copeland, A. Lahiri, and D. Wands, Phys. Rev. D 50, 4868 (1994). 